\title{
Conformal mappings of nearly quasi-Einstein manifolds
}

\author{
Füsun Özen Zengin and Bahar Kirik
}




\title{
CONFORMAL MAPPINGS OF NEARLY QUASI-EINSTEIN MANIFOLDS
}

\author{
FÜSUN ÖZEN ZENGIN AND BAHAR KIRIK
}

\begin{abstract}
In this paper, we consider a conformal mapping between two nearly quasi-Einstein manifolds $V_{n}$ and $\bar{V}_{n}$. We find some properties of this transformation from $V_{n}$ to $\bar{V}_{n}$ and some theorems are proved.
\end{abstract}

2000 Mathematics Subject Classification: 53B20; 53C25

Keywords: Nearly quasi-Einstein manifold, conformal mapping, conharmonic mapping, $\sigma$ (Ric)vector field

\section{INTRODUCTION}

A non-flat $n$-dimensional Riemannian or a semi-Riemannian manifold $(M, g)$, $(n>2)$ is said to be an Einstein manifold if the condition

$$
S(X, Y)=\frac{r}{n} g(X, Y)
$$

holds on $M$, where $S$ and $r$ denote the Ricci tensor and the scalar curvature of $(M, g)$, respectively. Einstein manifolds play an important role in Riemannian Geometry, as well as in general theory of relativity. For this reason, these manifolds have been studied by many authors.

A non-flat $n$-dimensional Riemannian manifold $(M, g),(n>2)$ is defined to be a quasi-Einstein manifold if its Ricci tensor $S$ of type $(0,2)$ is not identically zero and satisfies the following condition

$$
S(X, Y)=a g(X, Y)+b A(X) A(Y)
$$

where $a, b \in \mathbb{R}$ and $A$ is a non-zero 1 -form such that $g(X, U)=A(X)$ for all vector fields $X$ on $M$, [5]. Then $A$ is called the associated 1 -form and $U$ is called the generator of the manifold. Also M. C. Chaki and R. K. Maity [2] studied the quasiEinstein manifolds by considering $a$ and $b$ as scalars such that $b \neq 0$ and $U$ as a unit vector field.

A non-flat $n$-dimensional Riemannian manifold $(M, g),(n>2)$ is called a nearly quasi-Einstein manifold if its Ricci tensor $S$ of type $(0,2)$ is not identically zero and 
satisfies the following condition

$$
S(X, Y)=a g(X, Y)+b E(X, Y)
$$

where $a$ and $b$ are non-zero scalars and $E$ is a non-zero symmetric tensor of type $(0,2)$, [4]. Then $E$ is called the associated tensor and $a$ and $b$ are called the associated scalars of $M$. An $n$-dimensional nearly quasi-Einstein manifold is denoted by $N(Q E)_{n}$. An example of $N(Q E)_{4}$ has been given in [4].

Putting $X=Y=e_{i}$ in (1.3), we get

$$
r=n a+b \tilde{E}
$$

Here $r$ is the scalar curvature of $N(Q E)_{n}$ and $\tilde{E}=E\left(e_{i}, e_{i}\right)$ where $\left\{e_{i}\right\}, i=$ $1,2, \ldots, n$ is an orthonormal basis of the tangent space at each point of the manifold.

In this paper, we investigate a conformal mapping between two nearly quasiEinstein manifolds.

\section{CONFORMAL MAPPINGS OF NEARLY QUASI-EINSTEIN MANIFOLDS}

In this section, we suppose that $V_{n}$ and $\bar{V}_{n},(n>2)$ are two nearly quasi-Einstein manifolds with metrics $g$ and $\bar{g}$, respectively.

Definition 1. A conformal mapping is a diffeomorphism of $V_{n}$ onto $\bar{V}_{n}$ such that

$$
\bar{g}=e^{2 \sigma} g
$$

where $\sigma$ is a function on $V_{n}$. If $\sigma$ is constant, then it is called a homothetic mapping. In local coordinates, (2.1) is written as

$$
\bar{g}_{i j}(x)=e^{2 \sigma(x)} g_{i j}(x), \quad \bar{g}^{i j}=e^{-2 \sigma} g^{i j} .
$$

Besides those equations, we have the Christoffel symbols, the components of the curvature tensor, the Ricci tensor, and the scalar curvature, respectively

$$
\begin{aligned}
\bar{\Gamma}_{i j}^{h}= & \Gamma_{i j}^{h}+\delta_{i}^{h} \sigma_{j}+\delta_{j}^{h} \sigma_{i}-\sigma^{h} g_{i j}, \\
\bar{R}_{i j k}^{h}= & R_{i j k}^{h}+\delta_{k}^{h} \sigma_{i j}-\delta_{j}^{h} \sigma_{i k}+g^{h \alpha}\left(\sigma_{\alpha k} g_{i j}-\sigma_{\alpha j} g_{i k}\right) \\
& +\Delta_{1} \sigma\left(\delta_{k}^{h} g_{i j}-\delta_{j}^{h} g_{i k}\right), \\
\bar{S}_{i j}= & S_{i j}+(n-2) \sigma_{i j}+\left(\Delta_{2} \sigma+(n-2) \Delta_{1} \sigma\right) g_{i j}, \\
\bar{r}= & e^{-2 \sigma}\left(r+2(n-1) \Delta_{2} \sigma+(n-1)(n-2) \Delta_{1} \sigma\right),
\end{aligned}
$$

where $S_{i j}=R_{i j \alpha}^{\alpha}, r=S_{\alpha \beta} g^{\alpha \beta}, \sigma_{i}=\frac{\partial \sigma}{\partial x^{i}}=\nabla_{i} \sigma, \sigma^{h}=\sigma_{\alpha} g^{\alpha h}$ and

$$
\sigma_{i j}=\nabla_{j} \nabla_{i} \sigma-\nabla_{i} \sigma \nabla_{j} \sigma,
$$

$\Delta_{1} \sigma$ and $\Delta_{2} \sigma$ are the first and the second Beltrami's symbols which are determined by

$$
\Delta_{1} \sigma=g^{\alpha \beta} \nabla_{\alpha} \sigma \nabla_{\beta} \sigma, \quad \Delta_{2} \sigma=g^{\alpha \beta} \nabla_{\beta} \nabla_{\alpha} \sigma
$$


where $\nabla$ is the covariant derivative according to the Riemannian connection in $V_{n}$. We denote the objects of space conformally corresponding to $V_{n}$ by a bar, i. e., $\bar{V}_{n}$. If $\bar{V}_{n}$ is a $N(Q E)_{n}$, then we have, from (1.3), (2.2), and (2.5),

$$
\bar{b} \bar{E}_{i j}=b E_{i j}+(n-2) \sigma_{i j}+\left(\Delta_{2} \sigma+(n-2) \Delta_{1} \sigma+a-\bar{a} e^{2 \sigma}\right) g_{i j} .
$$

Definition 2. A vector field $\xi$ in a Riemannian manifold $M$ is called torse-forming if it satisfies the condition $\nabla_{X} \xi=\rho X+\phi(X) \xi$ where $X \in T M, \phi(X)$ is a linear form and $\rho$ is a function, [12]. In the local transcription, this reads

$$
\nabla_{i} \xi^{h}=\rho \delta_{i}^{h}+\xi^{h} \phi_{i}
$$

where $\xi^{h}$ and $\phi_{i}$ are the components of $\xi$ and $\phi$, and $\delta_{i}^{h}$ is the Kronecker symbol. A torse-forming vector field $\xi$ is called recurrent if $\rho=0$; concircular if the form $\phi_{i}$ is a gradient covector, i. e., there is a function $\psi(x)$ such that $\phi=d \psi(x)$; convergent, if it is concircular and $\rho=$ const $\cdot \exp (\psi)$.

Therefore, recurrent vector fields are characterized by the following equation

$$
\nabla_{X} \xi=\phi(X) \xi .
$$

Also, from the Definition 2., for a concircular vector field $\xi$, we get

$$
\left(\nabla_{Y} \xi\right) X=\rho g(X, Y)
$$

for all $X, Y \in T M$. A Riemannian space with a concircular vector field is called equidistant, $[10,11]$.

Conformal mappings of Riemannian spaces (or semi-Riemannian spaces) have been studied by many authors, $[1,3,6,9]$. In this section, we investigate the conformal mappings of nearly quasi-Einstein manifolds preserving the associated tensor $E$.

Theorem 1. If $V_{n}$ admits a conformal mapping preserving the associated tensor $E$ and the associated scalar $b$, then $V_{n}$ is an equidistant manifold.

Proof. Suppose that $V_{n}$ admits a conformal mapping preserving the associated tensor $E$ and the associated scalar $b$. Using (2.9), we obtain

$$
(n-2) \sigma_{i j}+\left(\beta+a-\bar{a} e^{2 \sigma}\right) g_{i j}=0
$$

where $\beta=\Delta_{2} \sigma+(n-2) \Delta_{1} \sigma$. In this case, we get

$$
\sigma_{i j}=\alpha g_{i j}
$$

where $\alpha=\frac{1}{n-2}\left(\bar{a} e^{2 \sigma}-a-\beta\right)$ is a function. Putting $\xi=-\exp (-\sigma)$ and using (2.7), (2.12) and (2.14), we get that $V_{n}$ is an equidistant manifold. Hence, the proof is complete. 
Theorem 2. An equidistant manifold $V_{n}$ admits a conformal mapping preserving the associated tensor $E$ if the associated scalars $\bar{a}$ and $\bar{b}$ satisfy both of the conditions

$$
\begin{aligned}
& \bar{b}=b, \\
& \bar{a}=e^{-2 \sigma}(a+\gamma),
\end{aligned}
$$

where $\gamma=\left(\frac{n-1}{n}\right)\left[2 \Delta_{2} \sigma+(n-2) \Delta_{1} \sigma\right]$.

Proof. Suppose that $V_{n}$ is an equidistant manifold. Then, there exists a concircular vector field $\xi$ satisfying the condition (2.12), that is, we have

$$
\nabla_{j} \xi_{i}=\rho g_{i j}
$$

where $\xi_{i} \equiv \nabla_{i} \xi$. Putting $\sigma=-\ln (-\xi(x))$ and using the condition (2.5), we obtain

$$
\bar{S}_{i j}=S_{i j}+\gamma g_{i j}
$$

where $\gamma=\left(\frac{n-1}{n}\right)\left[2 \Delta_{2} \sigma+(n-2) \Delta_{1} \sigma\right]$. Considering (1.3) in (2.16) and using (2.2), we get

$$
\bar{a} e^{2 \sigma} g_{i j}+\bar{b} \bar{E}_{i j}=(a+\gamma) g_{i j}+b E_{i j} .
$$

Taking $\bar{a}=e^{-2 \sigma}(a+\gamma)$ and $\bar{b}=b,(2.17)$ implies that $\bar{E}_{i j}=E_{i j}$. This completes the proof.

The conharmonic transformation is a conformal transformation preserving the harmonicity of a certain function. If the conformal mapping is also conharmonic, then we have, [8]

$$
\nabla_{i} \sigma^{i}+\frac{1}{2}(n-2) \sigma^{i} \sigma_{i}=0
$$

Theorem 3. Let $V_{n}$ be a conformal mapping with preservation of the associated tensor $E$ and the associated scalar $b$. A necessary and sufficient condition for this conformal mapping to be conharmonic is that the associated scalar $\bar{a}$ be transformed by $\bar{a}=e^{-2 \sigma} a$.

Proof. Suppose that $V_{n}$ admits a conformal mapping preserving the associated tensor $E$ and the associated scalar $b$. Using (2.7), (2.8) and (2.9), we obtain

$$
(n-2) \nabla_{j} \nabla_{i} \sigma-(n-2) \sigma_{i} \sigma_{j}+\left[\nabla_{h} \sigma^{h}+(n-2) \sigma^{h} \sigma_{h}+a-\bar{a} e^{2 \sigma}\right] g_{i j}=0 .
$$

Multiplying (2.19) by $g^{i j}$, we get

$$
\nabla_{h} \sigma^{h}+\frac{1}{2}(n-2) \sigma^{h} \sigma_{h}+\frac{n}{2(n-1)}\left(a-\bar{a} e^{2 \sigma}\right)=0 .
$$

If this mapping is conharmonic, using (2.18) in (2.20), we obtain $\bar{a}=e^{-2 \sigma} a$. The converse is also true. This completes the proof. 
Definition 3. A $\varphi$ (Ric)-vector field is a vector field on an $n$-dimensional Riemannian manifold $(M, g)$ and Levi-Civita connection $\nabla$, which satisfies the condition

$$
\nabla \varphi=\mu \mathrm{Ric}
$$

where $\mu$ is a constant and Ric is the Ricci tensor, [7]. When $(M, g)$ is an Einstein space, the vector field $\varphi$ is concircular. Moreover, when $\mu=0$, the vector field $\varphi$ is covariantly constant. In local coordinates, (2.21) can be written as

$$
\nabla_{j} \varphi_{i}=\mu S_{i j}
$$

where $S_{i j}$ denote the components of the Ricci tensor and $\varphi_{i}=\varphi^{\alpha} g_{i \alpha}$.

Suppose that $V_{n}$ admits a $\sigma($ Ric)-vector field. Then, we have

$$
\nabla_{j} \sigma_{i}=\mu S_{i j}
$$

where $\mu$ is a constant. Now, we can state the following theorem.

Theorem 4. Let us consider the conformal mapping (2.1) of a nearly quasiEinstein manifold $V_{n}$ with constant associated scalars being also conharmonic with the $\sigma$ (Ric)-vector field. A necessary and sufficient condition for the length of $\sigma$ to be constant is that the trace of the associated tensor $E$ of $V_{n}$ be constant.

Proof. We consider that the conformal mapping (2.1) of a nearly quasi-Einstein manifold $V_{n}$ admitting a $\sigma(\mathrm{Ric})$-vector field is also conharmonic. In this case, comparing (2.18) and (2.23), we get

$$
r=\frac{(2-n)}{2 \mu} \sigma^{i} \sigma_{i}
$$

where $r$ is the scalar curvature of $V_{n}$. If $V_{n}$ is of the constant associated scalars, from (1.4) and (2.24), we find

$$
\tilde{E}=\frac{1}{b}\left(\frac{(2-n)}{2 \mu} \sigma^{i} \sigma_{i}-n a\right) .
$$

If the length of $\sigma$ is constant, then $\sigma^{i} \sigma_{i}=c$, where $c$ is a constant. Thus, we can see that $\tilde{E}$ is constant. The converse is also true. Hence, the proof is complete.

\section{AN EXAMPLE OF A NEARLY QUASI-EINSTEIN MANIFOLD}

In this section, we consider a Riemannian metric $g$ on $\mathbb{R}^{4}$ by the formula

$$
d s^{2}=g_{i j} d x^{i} d x^{j}=\left(x^{4}\right)^{\frac{4}{3}}\left[\left(d x^{1}\right)^{2}+\left(d x^{2}\right)^{2}+\left(d x^{3}\right)^{2}\right]+\left(d x^{4}\right)^{2}
$$

where $i, j=1,2,3,4$ and $x^{1}, x^{2}, x^{3}, x^{4}$ are the standard coordinates of $\mathbb{R}^{4}$. Then the only non-vanishing components of the Christoffel symbols, the curvature tensor, 
the Ricci tensor and the scalar curvature are

$$
\begin{gathered}
\Gamma_{14}^{1}=\Gamma_{24}^{2}=\Gamma_{34}^{3}=\frac{2}{3 x^{4}}, \quad \Gamma_{11}^{4}=\Gamma_{22}^{4}=\Gamma_{33}^{4}=-\frac{2}{3}\left(x^{4}\right)^{\frac{1}{3}}, \\
R_{1441}=R_{2442}=R_{3443}=-\frac{2}{9\left(x^{4}\right)^{2 / 3}}, \\
R_{1221}=R_{1331}=R_{2332}=\frac{4}{9}\left(x^{4}\right)^{2 / 3}, \\
S_{11}=S_{22}=S_{33}=\frac{2}{3\left(x^{4}\right)^{2 / 3}}, \quad S_{44}=-\frac{2}{3\left(x^{4}\right)^{2}}, r=\frac{4}{3\left(x^{4}\right)^{2}} .
\end{gathered}
$$

Therefore $\mathbb{R}^{4}$ with the considered metric is a Riemannian manifold $\left(M_{4}, g\right)$ of nonvanishing scalar curvature. Let us now consider the associated scalars $a, b$, and the associated tensor $E$ as follows:

$$
a=-\frac{2}{3\left(x^{4}\right)^{2}}, \quad b=-\frac{1}{9 x^{4}}
$$

and

$$
E_{i j}(x)= \begin{cases}-12\left(x^{4}\right)^{1 / 3} & \text { for } i=j=1,2,3 \\ 0 & \text { for } i=j=4 \text { and } i \neq j\end{cases}
$$

at any point $x \in M$. To verify the relation (1.3), it is sufficient to check the relations $S_{i i}=a g_{i i}+b E_{i i}, i=1,2,3,4$ since for the other cases, (1.3) holds trivially. From (3.2), (3.3), and (3.4), we obtain

$$
\text { R.H.S of } S_{11}=a g_{11}+b E_{11}=\frac{2}{3\left(x^{4}\right)^{2 / 3}}=S_{11} \text {. }
$$

Similarly, $S_{22}, S_{33}$, and $S_{44}$ are also satisfied. Hence, $\left(M_{4}, g\right)$ endowed with the metric (3.1) is a $N(Q E)_{4}$ with the conditions (3.3) and (3.4).

Let $\left(M_{4}, g\right)$ endowed with the metric (3.1) be a conformal mapping with preservation of the associated tensor $E$ and the associated scalar $b$. Also, we choose $\sigma$ and $\bar{a}$ as follows:

$$
\sigma=\ln \left(x^{1} x^{2} x^{3}\right), \quad \bar{a}=-\frac{2}{3\left(x^{1} x^{2} x^{3} x^{4}\right)^{2}}
$$

where $x^{1}, x^{2}, x^{3}>0$. Now, we show that these choices satisfy Theorem 3 .

From (3.5), we get $\nabla_{i} \sigma=\frac{\partial \sigma}{\partial x^{i}}=\sigma_{i}=\frac{1}{x^{i}}$ for $i=1,2,3$ and $\sigma_{4}=0$. Moreover, the only non-vanishing covariant derivatives of $\sigma_{i}(i=1,2,3,4)$ are

$$
\begin{aligned}
& \nabla_{1} \sigma_{4}=\nabla_{4} \sigma_{1}=-\frac{2}{3 x^{1} x^{4}}, \\
& \nabla_{2} \sigma_{4}=\nabla_{4} \sigma_{2}=-\frac{2}{3 x^{2} x^{4}}, \\
& \nabla_{3} \sigma_{4}=\nabla_{4} \sigma_{3}=-\frac{2}{3 x^{3} x^{4}},
\end{aligned}
$$


and

$$
\nabla_{1} \sigma_{1}=-\frac{1}{\left(x^{1}\right)^{2}}, \quad \nabla_{2} \sigma_{2}=-\frac{1}{\left(x^{2}\right)^{2}}, \quad \nabla_{3} \sigma_{3}=-\frac{1}{\left(x^{3}\right)^{2}} .
$$

Using (3.6)-(3.9), we find

$$
g^{11} \nabla_{1} \sigma_{1}+g^{11} \sigma_{1} \sigma_{1}=0
$$

and similarly the other cases hold. Therefore, the condition (2.18) is satisfied.

Moreover, from (3.3) and (3.5), we obtain

$$
\bar{a} e^{2 \sigma}=-\frac{2}{3\left(x^{1} x^{2} x^{3} x^{4}\right)^{2}} \times e^{2 \ln \left(x^{1} x^{2} x^{3}\right)}=-\frac{2}{3\left(x^{4}\right)^{2}}=a .
$$

From (3.10) and (3.11), we see that the equation (2.20) is satisfied. Hence, Theorem 3 holds for $\left(M_{4}, g\right)$ endowed with the metric (3.1) and the conditions (3.3) and (3.5).

Now, we also show that $\left(M_{4}, g\right)$ endowed with the metric (3.1) is not a quasiEinstein manifold.

If possible, we have $S_{i j}=a g_{i j}+b A_{i} A_{j}$, where $i, j=1,2,3,4$. For $i=j$, we get from this relation

$$
S_{i i}=a g_{i i}+b A_{i} A_{i},
$$

for all $i=1,2,3,4$. Since $S_{i i} \neq 0$ and $g_{i i} \neq 0$, we can choose $a \neq 0, b \neq 0$ and $A_{i} \neq 0$ for all $i=1,2,3,4$ such that (3.12) holds. However, for these values of $a, b$ and $A_{i}$ and for $i \neq j$, the equation $S_{i j}=a g_{i j}+b A_{i} A_{j}$ cannot be satisfied because for $i \neq j, S_{i j}=g_{i j}=0$ but $A_{i} \neq 0$.

Therefore, $\left(M_{4}, g\right)$ is not a quasi-Einstein manifold. Thus, a $N(Q E)_{n}$ is not necessarily a quasi-Einstein manifold.

\section{REFERENCES}

[1] H. W. Brinkmann, "Einstein spaces which are mapped conformally on each other," Math. Ann., vol. 94, no. 1, pp. 119-145, 1925.

[2] M. C. Chaki and R. K. Maity, "On quasi-Einstein manifolds," Publ. Math. Debrecen, vol. 57, pp. 297-306, 2000.

[3] O. Chepurna, V. Kiosak, and J. Mikeš, "Conformal mappings of Riemannian spaces which preserve the Einstein tensor," Aplimat - Journal of Applied Mathematics, vol. 3, no. 1, pp. 253-258, 2010.

[4] U. C. De and A. K. Gazi, "On nearly quasi-Einstein manifolds," Novi Sad J. Math., vol. 38, no. 2, pp. 115-121, 2008.

[5] R. Deszcz, M. Glogowska, M. Hotlos, and Z. Senturk, "On certain quasi-Einstein semisymmetric hypersurfaces,” Annales Univ. Sci. Budapest., vol. 41, pp. 151-164, 1998.

[6] L. P. Eisenhart, Riemannian geometry. Princeton: Princeton Univ. Press, 1926.

[7] I. Hinterleitner and V. A. Kiosak, " $\phi$ (Ric)-vector fields in Riemannian spaces," Archivum Mathematicum, vol. 44, no. 5, pp. 385-390, 2008.

[8] Y. Ishii, “On conharmonic transformations," Tensor N. S., vol. 7, pp. 73-80, 1957.

[9] J. Mikeš, M. L. Gavrilchenko, and E. I. Gladysheva, "Conformal mappings onto Einstein spaces," Mosc. Univ. Math. Bull., vol. 49, no. 3, pp. 10-14, 1994. 
[10] N. S. Sinyukov, Geodesic mappings of Riemannian spaces. $\quad$ Moscow: Nauka, 1979.

[11] K. Yano, "Concircular geometry, I-IV," Proc. Imp. Acad., vol. 16, pp. 195-200, 354-360, 442448, 505-511, 1940.

[12] K. Yano, "On the torse-forming directions in Riemannian spaces," Proc. Imp. Acad., vol. 20, no. 6, pp. 340-345, 1944.

Authors' addresses

Füsun Özen Zengin

Istanbul Technical University, Faculty of Science and Letters, Department of Mathematics, Maslak, 34469 Istanbul, Turkey

E-mail address: fozen@itu.edu.tr

\section{Bahar Kirik}

Istanbul Technical University, Faculty of Science and Letters, Department of Mathematics, Maslak, 34469 Istanbul, Turkey

E-mail address: bkirikeitu.edu.tr 